\title{
A rare metastatic follicular thyroid carcinoma masquerading as primary sinonasal carcinoma presentation with unusual orbit, skull base and brain metastases
}

\author{
Case \\ Lim Iu Tong 1 , Baharudin Bin Abdullah', Avatar Singh A/L Mohan Singh ${ }^{3}$, Lina Ling \\ Report \\ Chooi $^{4}$, SuhanaBinti Abdul Rahim ${ }^{5}$, VijiA/L Ramasamy ${ }^{6}$ \\ 1,3,4,5,6 Department of Otorhinolaryngology, Head and Neck Surgery, Hospital Taiping, 34000, \\ Perak, ${ }^{2}$ Department of ORL-HNS, School of Medical Sciences, Health Campus USM, Kubang \\ Kerian, 16150, Kelantan, Malaysia.
}

\begin{abstract}
Tumour involving the sinonasal tract account for only $1 \%$ of all malignancies. The large majority of tumours affecting sinonasal tract are mainly primary neoplasm or from direct extension of adjacent region; metastatic tumour affecting sinonasal tract are extremely rare. Most of the metastases are originate from carcinoma inferior to the clavicle, including renal, breast, lung, and prostate. The incidence of metastases to sphenoid sinus are even rare. We describe a rare case of metastases of FTC to sinonasal tract, mimicking primary sinonasal carcinoma presentation, with unusual orbit, skull base and brain metastases.

A 66-year-old lady with history of subtotal thyroidectomy for FTC presented with epistaxis associated with proptosis of left eye. Nasal endoscopy revealed vascularised mass occupying the entire left nasal cavity. Endoscopic transnasal biopsy was performed which showed metastatic FTC with positive immunochemical staining for thyroglobulin, and thyroid transcription factor-1. Computed tomography angiography demostrated a large vascular tumour involving the left maxillary sinus, bilateral ethmoid and sphenoid sinuses. The mass causing erosion of nasal septum and pterygoid plates and left temporal bone, with extension to left orbit, infratemporal fossa and intracranial. Patient received thyroid stimulating hormone suppression therapy followed by radio-iodine ablation.

As surprisingly rare, the purpose of our study is to raise the awareness regarding the possibility of FTC metastatizing to sinonasal tract, orbit, skull base and intracranial. The completion thyroidectomy intervention is crucial for better future prognosis. Thyroid carcinoma should be considered in differential diagnosis of epistaxis and potential primary neoplasm in patient with orbital metastases.
\end{abstract}

Key Words: Differentiated thyroid cancer, follicular thyroid carcinoma, metastases, paranasal sinuses.

Received: 19 February 2020, Accepted: 31 January 2021

Corresponding Author: Lim Iu Tong, Department of Otorhinolaryngology, Head and Neck Surgery, Hospital Taiping, 34000, Perak, Malaysia, Tel.: 0127210249, E-mail: limiutong123@gmail.com

ISSN: 2090-0740, 2021

\section{INTRODUCTION}

Thyroid malignancies are one of the frequent encountered endocrine neck cancer and its incidence has been increasing for the past few decades, and approximately 0.5 to 10 cases per 100,000 people $^{[1]}$. Generally, thyroid cancer can be divided into 4 main groups namely, papillary, follicular, anaplastic, and medullary based on their histology differences. Amongst all the subtype, the second most frequent subtype is follicular thyroid cancer (FTC) following papillary subtype. Together, FTC and papillary are categorized as differentiated thyroid cancer (DTC), which contribute to 90 percent of all the thyroid carcinoma $^{[2,3]}$.
Tumour involving sinonasal tract are account for only $1 \%$ of all malignancies, and of these, only a handful of cases are metastases ${ }^{[4]}$. The large majority of tumours involving the paranasal sinuses (PNS) are mainly from its primary site or direct extension from adjacent anatomical regions, metastases to the nose and PNS are extremely rare, and most of the metastases are originate from the carcinoma inferior to clavicle ${ }^{[5]}$. Sachmenchi et al., 2014 point out that only 12 cases of metastases from thyroid carcinoma to sphenoid sinuses reported to date in the literature, and of these, half of the cases are from $\mathrm{FTC}^{[6]}$. Har-El et al. and Weig et al. also found that sphenoid sinus is an extremely rare area for the deposition of metastatic carcinoma ${ }^{[7,8]}$.

A literature review by Jun Shen et al. in 2015, there are 
only 28 documented cases of skull base metastases from differentiated thyroid carcinoma have been reported ${ }^{[9]}$. Daumerie et al. concluded that metastatic deposition of the orbit from thyroid malignancies are relatively rare with limited number of isolated case reported in literature ${ }^{[10]}$. Goldberg et al. described the frequent primary sites tumour that responsible for the orbital metastases including breast $(42 \%)$, followed by lung $(11 \%)$ and prostate $(8.3 \%)^{[11]}$. According to Altmari et al., the incidence of distant metastases from FTC to brain are rare as well, at a frequency about $1 \%$ overall $^{[12]}$. Fujita, 2009 presented the metastatic deposits from FTC to the brain are quite rare with less than 10 cases have been reported instances ${ }^{[13]}$.

In this article, we have the opportunity to share the most interesting rare case of metastases of FTC to sinonasal tract, mimicking primary sinonasal carcinoma presentation, with unusual orbit, skull base and brain metastases.

\section{INTRODUCTION}

A 66-year old Indian lady presented to us with a 2 days history of bilateral epistaxis and progressive bilateral nasal obstruction for few months duration. She reported having subtotal thyroidectomy done 15 years previously in the private hospital for FTC but no further details available. She was told to be free of disease during her last consultation 12 years ago. She denied numbness over the face, and there was no history of trauma to the nasal region and she do not have intracranial symptoms.

On general examination, her nasal framework appeared intact. We noticed mild proptosis on the left eyes, but did not affect her visual acuity and ocular movement (Figure 1). The cervical examination show no abnormalities or any swelling except a well healed thyroidectomy scar. Neurological examination was unremarkable. Zero degree nasal endoscopy assessment in clinic revealed a huge vascularised mass occupying the entire left nasal cavity and posterior choanae of right nasal cavity (Figure 2). The thyroid function test was within normal limits.

Computed tomography (CT) neck with CT angiography demonstrated a large heterogenous enhancing mass measuring $5.1 \times 5.1 \times 5.0 \mathrm{~cm}$ causing erosion of nasal septum, erosion of pterygoid plates, erosion of left temporal bone and left orbital floor, with the involvement of left maxillary sinus, bilateral ethmoid, bilateral sphenoid sinuses and extension of tumour into the left infratemporal fossa, left temporal lobe and left orbit and causing proptosis on the left side. (Figure 3). Residual small right thyroid lobe with multiple small hypodense nodules $0.4 \times 0.3 \mathrm{~cm}$ were noted. Therefore patient was proceeded with ultrasound guided fine needle aspiration of cytology over right thyroid, however the results showed benign follicular nodule with evidence of cystic degeneration.

Patient was taken to operating theatre and we performed a diagnostic endoscopic transnasal biopsy of the vascularized mass which occupying the whole left nasal cavity and posterior choanae of the right under general anesthesia. Pathological evaluation showed malignant epithelial cells disposed in micro and normofollicular pattern, which contain eosinophilic colloid material. The individual malignant cells exhibit mild to moderate nuclear pleomorphism, mitosis and inconspicuous nucleoli. Immunochemical staining for thyroglobulin, and thyroid transcription factor-1 (TTF-1) were strongly positive and consistent with FTC.

Patient was referred to oncology center in Penang Hospital for further management. She received thyroid stimulating hormone suppression therapy followed by radioactive iodine (RAI) ablation in oncology center.

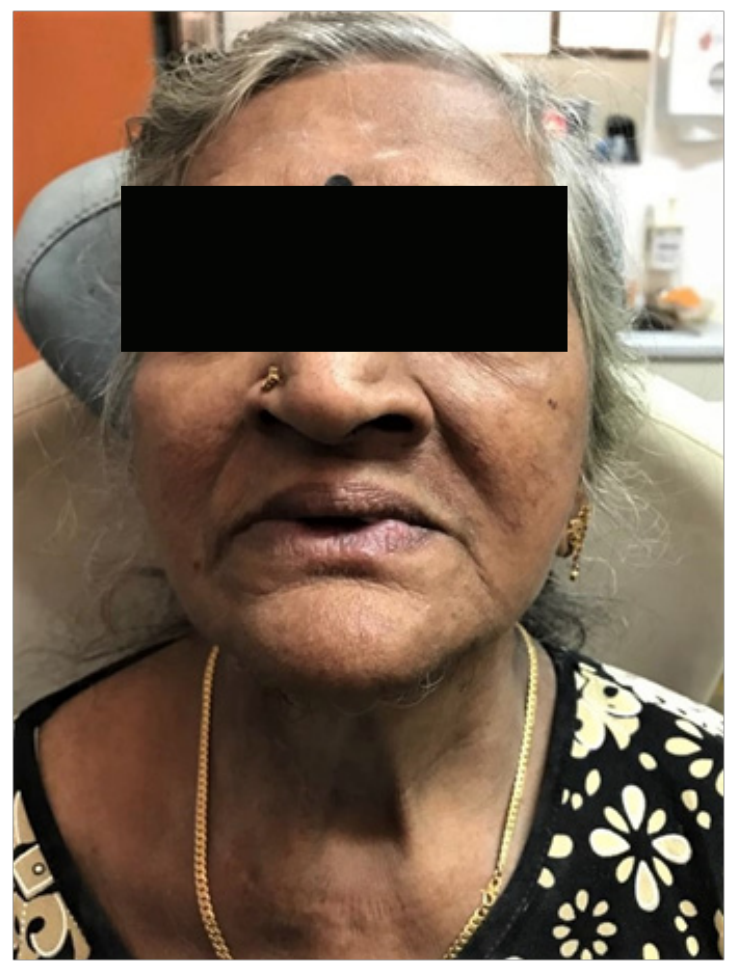

Fig. 1: Clinical photograph showing left proptosis with well healed previous thyroidectomy scar.

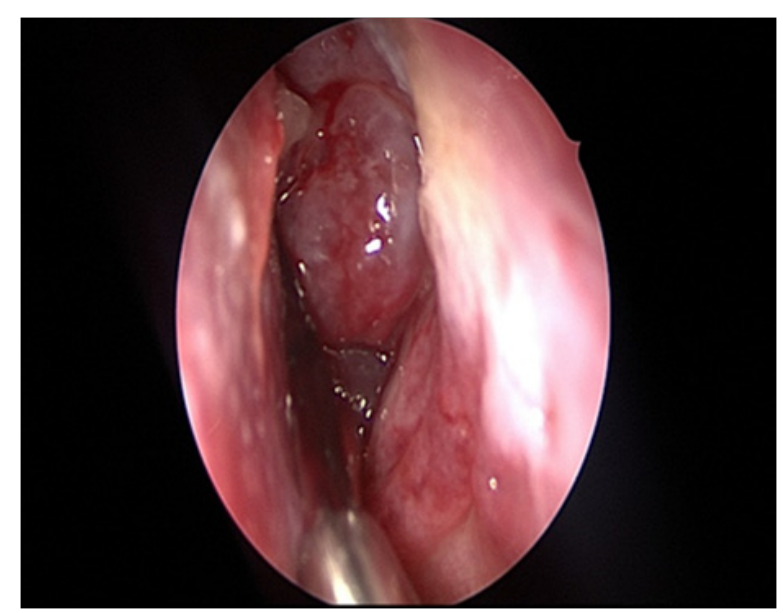

Fig. 2: Nasal endoscopy assessment revealed a huge vascularised mass occupying the left nasal cavity. 

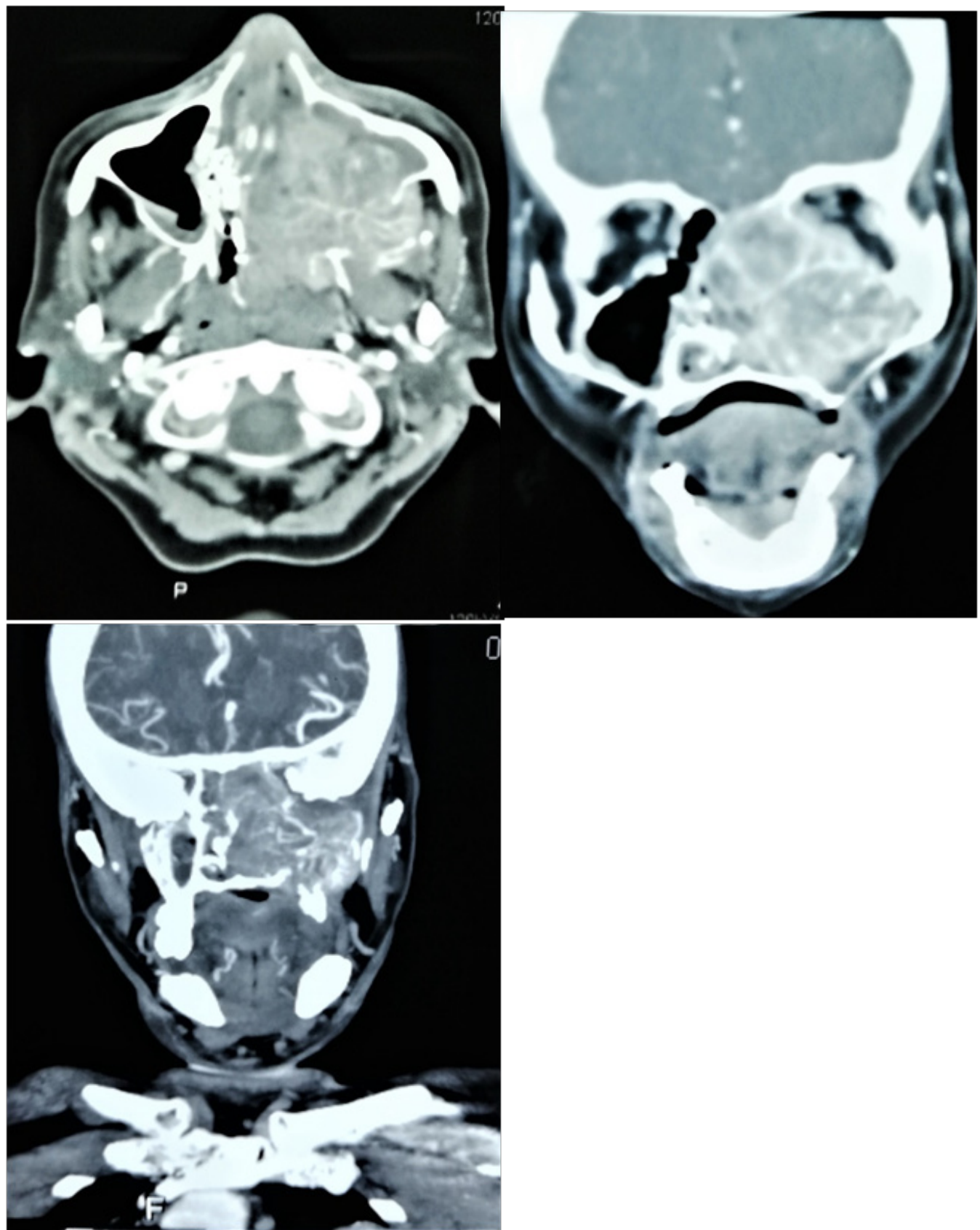

Fig. 3: Evaluation with CT neck with CT Angiography demonstrated a large heterogenous enhancing mass measuring $5.1 \times 5.1 \times 5.0 \mathrm{~cm}$ causing erosion of nasal septum, erosion of pterygoid plates, erosion of left temporal bone and left orbital floor, with the involvement of left maxillary sinus, bilateral ethmoid, bilateral sphenoid sinuses and extension of tumour into the left infratemporal fossa, left temporal lobe and left orbit and causing proptosis on the left side as visualized in axial view (A) and coronal view (B, C).

\section{DISCUSSION}

Amongst this well differentiated carcinomas, papillary accounts for $80-85 \%$, follicular $10-15 \%$, and Hurthle cell carcinoma $3-5 \%{ }^{[14]}$. In comparison with papillary thyroid carcinoma (PTC), FTC tends to occur in elderly patient, spreading though the blood stream and display in a more aggressive manner ${ }^{[15]}$. DTC metastases to PNS is fairly uncommon. Amongst the various PNS, Carlos et al, 2009 and Berstein et al., 1979, both described the maxillary sinuses are the most commonly affected PNS by metastatic foci, followed in frequency by the lesion in ethmoid, frontal and the less frequent one, which is sphenoid sinus ${ }^{[16,17]}$. Barrs et al. reviewed a series of 8 patients with metastases to PNS, showed 2 patients with primary tumour was in the prostate, followed by another 2 patients had myeloma, 1 patient each with lung, breast, kidney and thyroid (follicular) ${ }^{[18]}$. According to Mickel et al., metastases to PNS is possible to occur from any potential primary tumour origins in human 
body, in their order, from renal, breast, prostate, to lung carcinoma, are outlined as the most frequent primaries in the literature ${ }^{[19]}$.

In this case, our patient presented to us with nasal complaints of epistaxis and nasal obstruction, and there was no significant symptoms related to the primary thyroid problems. Patient's history and the related nasal scope findings are leading us to the initial diagnosis of sinonasal malignancies as the clinical presentation is similar to those presented with primary tumour in the sinonasal area. Therefore, it is very important for the attending surgeon to have a high index of suspicion and always evaluate the possibility of metastatic tumour from the previous treated cancerous site to prevent any delayed in diagnosis. Yamasoba et al reported that epistaxis was the most frequent initial sign of metastatic tumour to sinonasal tract due to the hypervascular nature of thyroid carcinoma itself. Other nonspecific symptoms such as headache, facial pain, visual disturbance and nasal obstructions also been reported $^{[20]}$

Regarding the aetiopathogenesis spreading of thyroid cancer into the PNS and skull base, Batson postulated that vertebral venous plexus, also known as Batson plexus, which composed a valveless venous system permit communication from skull to pelvic region, and thus responsible for the hematogenous spreading of cancer cells toward the head and neck structures, including PNS ${ }^{[21]}$. Metastases occur in the maxillary sinus are more common as compared to others sinuses due to the greater artery supply in this sinus by the branches from external carotid artery. Lymphatic route is an alternative pathway, enable the retrograde flow of tiny tumour emboli to move from the thyroid gland to the skull base and PNS site ${ }^{[22,23]}$.

CT scan is the good modality for delineating the nature of lesion, bony involvement and erosions from metastatic tumour toward PNS, but Magnetic Resonance Imaging is more superior for the assessment of soft tissue involvement, the true extent and defining the neighbouring structures better. As the metastases lesion to the skull base and PNS can be very vascularity in nature, angiography and embolization should be considered for pre-operative planning if the lesion is operable, and also opted for bleeding control via embolization if palliative option offered $^{[24]}$. However, imaging studies unable to ascertain and differentiate the primary tumour from the metastatic lesions, and the only ultimate method needed to establish the diagnosis is still depend on the pathological findings.

Clark analyzed 51 patients with DTC who had less than total thyroidectomy performed, $26(51 \%)$ had cancer remained in the remnant of thyroid tissue, and $5(10 \%)$ have focal cancer in the contralateral thyroid lobe to the one operated ${ }^{[25]}$. Ernest, 1999 postulated that the best management for DTC is near total thyroidectomy followed by radio-iodine ablation of thyroid remnant, as it can minimize the recurrence and enhance the survival rate ${ }^{[26]}$ Ernest also suggest that, completion thyroidectomy should be proceed if only partial lobectomy was performed for DTC initially, and for tumour sized $1.5 \mathrm{~cm}$ or bigger, so that can avoid the high chances of residual tumour which may occur in the opposite thyroid lobe ${ }^{[26]}$. Our patient had subtotal thyroidectomy done 15 years back, without RAI and completion thyroidectomy intervention, and this can explained the possibility of the remnant thyroid tissue giving rise to the current metastases issues.

\section{CONCLUSION}

In conclusion, metastases of DTC to PNS is extremely rare and even rarer to the sphenoid sinus. Though exceedingly rare, the metastatic lesion might be a late feature and the only expression of the primary carcinoma. Therefore, attending surgeon should always considered the possibility of sinonasal metastases as one of the differential diagnosis of epistaxis.

\section{KEY MESSAGE}

Though thyroid lobectomy was adequate for small solitary thyroid tumour, we believed total or near total thyroidectomy especially for lesion $1.5 \mathrm{~cm}$ or greater followed by RAI treatment will reduce the recurrence rate, prevent metastases possibility and therefore improve the patient survical chances.

\section{ACKNOWLEDGEMENT}

I would like to express my special thanks of gratitude to Professor Baharuddin, Dr Lina Ling Chooi, Dr Avatar Singh, DrSuhana and Dr Viji for their guidance and support in completing my case report.

\section{REFERENCES}

1. Ferrer JG, Merino JT, Ponce JM, Piñón FS, editors. Unusual metastasis of differentiated thyroid carcinoma. Anales de medicina interna (Madrid, Spain: 1984); 2002.

2. Cooper DS, Doherty GM, Haugen BR, Kloos RT, Lee SL, Mandel SJ, et al. Revised American Thyroid Association management guidelines for patients with thyroid nodules and differentiated thyroid cancer: the American Thyroid Association (ATA) guidelines taskforce on thyroid nodules and differentiated thyroid cancer. Thyroid. 2009;19(11):1167-214.

3. Jasim S, Ozsari L, Habra MA. Multikinase inhibitors use in differentiated thyroid carcinoma. Biologics: targets and therapy. 2014;8:281. 
4. Tufano RP, Mokadam NA, Montone KT, Weinstein GS, Chalian AA, Wolf PF, et al. Malignant tumors of the nose and paranasal sinuses: hospital of the University of Pennsylvania experience 1990-1997. American journal of rhinology. 1999;13(2):117-24.

5. Zachariades N. Neoplasms metastatic to the mouth, jaws and surrounding tissues. Journal of CranioMaxillofacial Surgery. 1989;17(6):283-90.

6. Sachmechi I, Bitton RN, Sanelli-Russo S, Thongpooswan S. An aggressive follicular variant of papillary thyroid carcinoma with unusual metastases A case report and review of literature. British Journal of Medical Practitioners. 2014;7:25-8.

7. Har-El G, Avidor I, Weisbord A, Sidi J. Carcinoma of the prostate metastatic to the maxillary antrum. Head and neck surgery. 1987;10(1):55-8.

8. Weig S, Howard JJ, Fry T. Metastatic laryngeal carcinoma as a cause of progressive ophthalmoplegia. Southern medical journal. 1989;82(8):1051-2.

9. Shen J, Wang $S$, Zhao $X$, Shao $X$, Jiang $X$, Dai Y, et al. Skull metastasis from follicular thyroid carcinoma: report of three cases and review of literature. Int J Clin Exp Pathol. 2015;8(11):15285-93.

10. Daumerie C, De Potter P, Godfraind C, Rahier J, Jamar F, Squifflet J-P. Orbital metastasis as primary manifestation of thyroid carcinoma. Thyroid. 2000;10(2):189-92.

11. Goldberg RA, Rootman J. Clinical characteristics of metastatic orbital tumors. Ophthalmology. 1990;97(5):620-4

12. Altimari-Romero RA, Montenegro FL, Michaluart Junior P, De Carlucci D, Jr., Medina-Santos LR, Ferraz AR. [Brain metastases from papillary thyroid carcinoma: a case report and review of the literature]. Revista do Hospital das Clinicas. 1997;52(5):263-6.

13. Fujita T, Ogasawara Y, Doihara H. Solitary cranial metastasis of thyroid carcinoma 13 years after primary surgery: report of a case. Surgery today. 2009;39(1):44-7.

14. Jossart GH, Clark OH. Well-differentiated thyroid cancer. Current problems in surgery. 1994;31(12):933-1012.

15. Baloch ZW, Livolsi VA. Pathology of thyroid and parathyroid disease. Sternberg's diagnostic surgical pathology. 2004;4:558-619.

16. Bernstein JM, Montgomer WW, Balogh Jr K Metastatic tumors to the maxilla, nose, and paranasal sinuses. The laryngoscope. 1966;76(4):621-50.

17. Duque-Fisher CS, Casiano R, Vélez-Hoyos A, Londoño-Bustamente AF. Metastasis to the sinonasal region. Acta Otorrinolaringologica (English Edition). 2009;60(6):428-31.

18. Barrs DM, McDonald TJ, Whisnant JP. Metastatic tumors to the sphenoid sinus. The Laryngoscope. 1979;89(8):1239-43.

19. Mickel RA, Zimmerman MC. The sphenoid sinus-a site for metastasis. Otolaryngology - Head and Neck Surgery. 1990;102(6):709-16.

20. Yamasoba T, Kikuchi S, Sugasawa M, Higo R, Sasaki T. Occult follicular carcinoma metastasizing to the sinonasal tract. ORL. 1994;56(4):239-43.

21. Batson OV. The function of the vertebral veins and their role in the spread of metastases. Annals of surgery. 1940;112(1):138.

22. Gottlieb MD, Roland Jr JT. Paradoxical spread of renal cell carcinoma to the head and neck. The Laryngoscope. 1998;108(9):1301-5.

23. Huang H-H, Chang P-H, Fang T-J. Sinonasal metastatic hepatocellular carcinoma. American journal of otolaryngology. 2007;28(4):238.

24. López F, Devaney KO, Hanna EY, Rinaldo A, Ferlito A. Metastases to nasal cavity and paranasal sinuses. Head and neck. 2016;38(12):1847-54.

25. Clark OH. Total thyroidectomy: the treatment of choice for patients with differentiated thyroid cancer. Annals of Surgery. 1982;196(3):361.

26. MAZZAFERRI EL. An overview of the management of papillary and follicular thyroid carcinoma. Thyroid. 1999;9(5):421-7. 